\title{
Local bulk physics from intersecting modular Hamiltonians
}

\author{
Daniel Kabat ${ }^{a}$ and Gilad Lifschytz ${ }^{b}$ \\ ${ }^{a}$ Department of Physics and Astronomy, Lehman College, City University of New York, \\ Bronx NY 10468, U.S.A. \\ ${ }^{b}$ Department of Mathematics and Haifa Research Center for Theoretical Physics and Astrophysics, \\ University of Haifa, Haifa 31905, Israel \\ E-mail: daniel.kabat@lehman.cuny.edu, giladl@research.haifa.ac.il
}

ABSTRACT: We show that bulk quantities localized on a minimal surface homologous to a boundary region correspond in the CFT to operators that commute with the modular Hamiltonian associated with the boundary region. If two such minimal surfaces intersect at a point in the bulk then CFT operators which commute with both extended modular Hamiltonians must be localized at the intersection point. We use this to construct local bulk operators purely from CFT considerations, without knowing the bulk metric, using intersecting modular Hamiltonians. For conformal field theories at zero and finite temperature the appropriate modular Hamiltonians are known explicitly and we recover known expressions for local bulk observables.

KeYworDS: AdS-CFT Correspondence, Conformal Field Theory

ARXIV EPRINT: 1703.06523 


\section{Contents}

1 Introduction 1

2 Modular Hamiltonian in $\mathbf{A d S}_{3} \quad 3$

2.1 CFT quantities invariant under the modular Hamiltonian 5

2.2 Extended modular Hamiltonian $\quad 7$

$\begin{array}{llr}2.3 & \text { Finite temperature } & 9\end{array}$

3 Bulk operators from intersecting modular Hamiltonians 10

$\begin{array}{ll}3.1 \text { Recovering smearing functions for the vacuum state } & 11\end{array}$

$\begin{array}{lll}3.1 .1 & \text { Derivative representation } & 13\end{array}$

$\begin{array}{lll}3.2 & \text { Recovering bulk modes } & 14\end{array}$

$\begin{array}{lll}3.3 & \text { Time dependence } & 15\end{array}$

$\begin{array}{ll}3.4 & \text { Recovering BTZ bulk operators } \\ \end{array}$

4 Conclusions $\quad 19$

$\begin{array}{ll}\text { A Extended modular Hamiltonian } & 20\end{array}$

B Action of $\tilde{H}_{\text {mod }}$ on operators off the RT surface 20

$\begin{array}{ll}\text { C Geodesics in BTZ } & 21\end{array}$

\section{Introduction}

Having a procedure for recovering bulk physics from the CFT is fundamental to our quest to understand quantum gravity using AdS/CFT [1]. The Ryu-Takayanagi (RT) relation [2] between the area of minimal surfaces anchored on the boundary (RT surfaces) and entanglement entropy in the CFT is one example of how bulk quantities can be computed from the CFT. The first law of entanglement entropy has been used together with the RT and HRT formulas [3] to derive the linearized Einstein equations in the bulk [4].

Natural bulk objects to consider are bulk field operators. Traditionally the program of constructing bulk field operators in the CFT starts from knowledge of the bulk metric. This information is used to compute smearing functions which provide the leading-order expression (in $1 / N$ ) for a local bulk field in terms of a single-trace primary scalar operator in the CFT [5-7].

$$
\Phi^{(0)}(x, z)=\int d x^{\prime} K\left(x, z \mid x^{\prime}\right) \mathcal{O}\left(x^{\prime}\right)
$$

$\Phi^{(0)}$ is a CFT operator which reproduces the correct bulk 2-point function when inserted in a CFT correlator. However the expression for $\Phi^{(0)}$ is not unique. Among the expressions 
we will be using are the complex coordinate representation (for Poincaré coordinates in $\left.\mathrm{AdS}_{3}\right)[8]$

$$
\Phi^{(0)}(Z, X, T)=\frac{\Delta-1}{\pi} \int_{y^{\prime 2}+t^{\prime 2}<Z^{2}} d t^{\prime} d y^{\prime}\left(\frac{Z^{2}-y^{\prime 2}-t^{\prime 2}}{Z}\right)^{\Delta-2} \mathcal{O}\left(T+t^{\prime}, X+i y^{\prime}\right)
$$

and the representation in terms of mode functions [9].

$$
\Phi^{(0)}(Z, X, T)=\frac{2^{\Delta} \Gamma(\Delta)}{8 \pi^{2}} \int_{|\omega|>|k|} d \omega d k e^{-i \omega T} e^{i k X} Z\left(\omega^{2}-k^{2}\right)^{-\nu / 2} J_{\nu}\left(Z \sqrt{\omega^{2}-k^{2}}\right) \mathcal{O}(\omega, k)
$$

$1 / N$ corrections to these expressions - for example to define CFT operators that will reproduce the expected bulk 3-point functions - can be obtained purely from the CFT using bulk locality as a guiding principle. Imposing bulk microcausality corresponds to canceling unwanted singularities in correlation functions. To achieve this it is necessary to correct the definition of the bulk field by adding to $\Phi^{(0)}$ an infinite tower of higher-dimension multi-trace operators. To restore locality in $n$-point functions it is necessary to add CFT operators involving up to $n-1$ traces, all smeared with the appropriate smearing functions as in (1.1). In this way the expressions relevant to bulk 3-point functions were obtained in $[10,11]$, and the resulting bulk fields were shown to obey the correct bulk equations of motion. The expressions needed to reproduce bulk 4-point functions were obtained in [12] with the help of crossing symmetry. These results extend with some modifications to bulk fields with spin [13-16]. However throughout this program the starting point, that is the lowest-order smearing functions, were computed using knowledge of the bulk metric. ${ }^{1}$

In this paper we obtain the zeroth-order bulk operator $\Phi^{(0)}$, up to a multiplicative coefficient, ${ }^{2}$ purely from CFT considerations. The basic idea is that the modular Hamiltonian associated to a boundary region has the same action on bulk quantities as the associated bulk modular Hamiltonian [19]. The RT surface (the minimal bulk surface homologous to the boundary region) plays the role of a bifurcation surface in Rindler coordinates (the surface where the past and future Rindler horizons intersect). Just as the bifurcation surface is invariant under Rindler time evolution, the RT surface is invariant under the action of the bulk modular Hamiltonian. This means CFT objects which are localized in the bulk on an RT surface should commute with the corresponding boundary modular Hamiltonian.

To proceed it's convenient to define an extended modular Hamiltonian which generates a non-trivial flow everywhere in the bulk except on the RT surface. If a collection of RT surfaces intersect at a point in the bulk, then CFT quantities which commute with all of the corresponding extended modular Hamiltonians must be localized at the intersection point. We can impose this as a condition to construct local observables in the bulk. The construction is simplest in the case of 2-D CFT where RT surfaces are just lines.

Recently $\Phi^{(0)}$ has been shown to be related to several natural CFT objects. In [20$22]$ the integral of $\Phi^{(0)}$ over an RT surface was found to be related to a conformal block

\footnotetext{
${ }^{1}$ An alternative construction for empty AdS is based on representation theory $[17,18]$.

${ }^{2}$ The requirement of locality only fixes bulk observables up to a multiplicative coefficient. The coefficient could be chosen to depend on bulk space-time position. We will discuss this ambiguity more in section 3 .
} 
operator. $^{3}$ In [24-26] it was shown that $\Phi^{(0)}$ creates a boundary cross-cap state in the CFT. One of the motivations of the present paper was to understand the relation between these different descriptions of $\Phi^{(0)}$. The construction developed here makes the connection much clearer.

An outline of this paper is as follows. In section 2 we review the modular Hamiltonian appropriate to a segment of the boundary and extend it outside this region in a natural way to obtain what we call the extended modular Hamiltonian. We use this to show that bulk operators on the RT surface commute with the modular Hamiltonian in a variety of contexts. In section 3 we turn the argument around and search for a CFT operator that commutes with the extended modular Hamiltonians associated with two different boundary segments. Provided the corresponding RT surfaces intersect we explicitly solve this condition and find the correct smearing function for a local operator that lives on the intersection. We do this for the vacuum state of the CFT on a line in three ways: using complexified coordinates, using a derivative expansion, and in momentum space. The latter recovers the Poincaré mode expansion of a bulk field from the CFT. We also do this for a CFT on a line at finite temperature and recover the complexified smearing function for a BTZ black hole. In appendix A we establish the relationship between conventional and extended modular Hamiltonians, in appendix B we show that the extended modular Hamiltonian has the same action on bulk operators which are off the RT surface as the bulk Rindler Hamiltonian, and in appendix $\mathrm{C}$ we study geodesics in BTZ. For convenience in all explicit computations we specialize to $\mathrm{AdS}_{3} / \mathrm{CFT}_{2}$.

\section{Modular Hamiltonian in $\mathrm{AdS}_{3}$}

We work in $\mathrm{AdS}_{3} / \mathrm{CFT}_{2}$ in Poincaré coordinates. The bulk metric is

$$
d s^{2}=\frac{l^{2}}{Z^{2}}\left(-d T^{2}+d Z^{2}+d X^{2}\right)
$$

and the CFT metric is

$$
d s^{2}=-d T^{2}+d X^{2}
$$

We introduce boundary light-front coordinates

$$
\xi=X-T, \quad \bar{\xi}=X+T
$$

A space-like segment in a $(1+1)$ dimensional CFT defines a causal diamond based on the segment. The diamond $D(x, y)$ is defined through its upper tip $y^{\mu}$ and its lower tip $x^{\mu}$, which we describe using the light-front coordinates

$$
(u, \bar{u})=\left(x^{1}-x^{0}, x^{1}+x^{0}\right), \quad(v, \bar{v})=\left(y^{1}-y^{0}, y^{1}+y^{0}\right)
$$

If we choose a diamond on the boundary whose left and right tips lie on the $T=0$ slice at points $y_{1}$ and $y_{2}$ then

$$
y^{\mu}=\left(\frac{y_{2}-y_{1}}{2}, \frac{y_{2}+y_{1}}{2}\right) \quad x^{\mu}=\left(\frac{y_{1}-y_{2}}{2}, \frac{y_{2}+y_{1}}{2}\right)
$$

\footnotetext{
${ }^{3}$ There is also a connection with geodesic Witten diagrams [23].
} 
so that

$$
u=y_{2}, \quad v=y_{1}, \quad \bar{u}=y_{1}, \quad \bar{v}=y_{2} .
$$

For a CFT in its vacuum state the modular Hamiltonian can be written explicitly [27].

$$
H_{\text {mod }}=2 \pi \int_{v}^{u} d \xi \frac{(u-\xi)(\xi-v)}{u-v} T_{\xi \xi}(\xi)+2 \pi \int_{\bar{u}}^{\bar{v}} d \bar{\xi} \frac{(\bar{v}-\bar{\xi})(\bar{\xi}-\bar{u})}{\bar{v}-\bar{u}} \bar{T}_{\bar{\xi} \bar{\xi}}(\bar{\xi})
$$

We define coordinates $\eta$ and $\bar{\eta}$ by

$$
\begin{aligned}
& d \eta=\frac{(u-v)}{(u-\xi)(\xi-v)} d \xi \\
& d \bar{\eta}=\frac{(\bar{v}-\bar{u})}{(\bar{v}-\bar{\xi})(\bar{\xi}-\bar{u})} d \bar{\xi}
\end{aligned}
$$

which are solved by

$$
\begin{aligned}
e^{\eta} & =\frac{\xi-v}{u-\xi} \\
e^{\bar{\eta}} & =\frac{\bar{\xi}-\bar{u}}{\bar{v}-\bar{\xi}}
\end{aligned}
$$

These Rindler-like null coordinates $(\eta, \bar{\eta})$ cover the diamond. In terms of dimensionless time and space coordinates

$$
\eta=\phi-\hat{t}, \quad \bar{\eta}=\phi+\hat{t}
$$

the diamond is covered by $-\infty<\hat{t}, \phi<\infty$.

Under the change of coordinates $\xi \rightarrow \eta$ and $\bar{\xi} \rightarrow \bar{\eta}$ we have ${ }^{4}$

$$
\begin{aligned}
& T_{\eta \eta}(\eta)=\left(\frac{d \xi}{d \eta}\right)^{2} T_{\xi \xi}-\frac{c}{24 \pi} S(\xi, \eta) \\
& T_{\bar{\eta} \bar{\eta}}(\bar{\eta})=\left(\frac{d \bar{\xi}}{d \bar{\eta}}\right)^{2} T_{\bar{\xi} \bar{\xi}}-\frac{\bar{c}}{24 \pi} S(\bar{\xi}, \bar{\eta})
\end{aligned}
$$

where

$$
S(\xi, \eta)=\frac{\frac{d \xi}{d \eta} \frac{d^{3} \xi}{d \eta^{3}}-\frac{3}{2}\left(\frac{d^{2} \xi}{d \eta^{2}}\right)^{2}}{\left(\frac{d \xi}{d \eta}\right)^{2}}
$$

For the change of coordinates in $(2.8)$ one finds $S(\xi, \eta)=S(\bar{\xi}, \bar{\eta})=-\frac{1}{2}$. Thus we can write the modular Hamiltonian (2.7) as

$H_{\text {mod }}=2 \pi \int_{-\infty}^{\infty} d \phi\left(T_{\eta \eta}(\phi)+T_{\bar{\eta} \bar{\eta}}(\phi)\right)-\frac{c}{24} \int_{y_{1}}^{y_{2}} d \xi \frac{y_{2}-y_{1}}{\left(y_{2}-\xi\right)\left(\xi-y_{1}\right)}-\frac{\bar{c}}{24} \int_{y_{1}}^{y_{2}} d \bar{\xi} \frac{y_{2}-y_{1}}{\left(y_{2}-\bar{\xi}\right)\left(\bar{\xi}-y_{1}\right)}$

In Poincaré coordinates a bulk geodesic $\gamma$ on the $T=0$ slice connecting the points $y_{1}$ and $y_{2}$ on the boundary is given by the semicircle

$$
\left(X-y_{1}\right)\left(y_{2}-X\right)=Z^{2}
$$

\footnotetext{
${ }^{4}$ This is more commonly written in terms of $T(\xi)=-2 \pi T_{\xi \xi}$ and $\bar{T}(\bar{\xi})=-2 \pi T_{\bar{\xi} \bar{\xi}}$.
} 
Proper length along this geodesic is

$$
d s=\frac{l}{2} \frac{y_{2}-y_{1}}{\left(X-y_{1}\right)\left(y_{2}-X\right)} d X
$$

Using this and $c=\bar{c}=\frac{3 l}{2 G}$ the last two terms in (2.13) can be seen to be

$$
-\frac{1}{4 G} \int_{\gamma} d s
$$

This is just the RT term, i.e. the area of the minimal surface. So in fact

$$
2 \pi \int_{-\infty}^{\infty} d \phi\left(T_{\eta \eta}(\phi)+T_{\bar{\eta} \bar{\eta}}(\phi)\right)=H_{\mathrm{mod}}+\frac{A}{4 G}
$$

The authors of [19] identified the left-hand side of (2.17) with the boundary modular Hamiltonian and interpreted $H_{\text {mod }}$ as a bulk modular Hamiltonian which generates bulk time evolution in the appropriate bulk Rindler wedge plus fluctuations of the RT surface (see also [28]). The computations of this paper will, among other things, confirm that $H_{\text {mod }}$ acts on CFT operators which represent bulk quantities in the manner expected for a bulk Rindler Hamiltonian.

\subsection{CFT quantities invariant under the modular Hamiltonian}

We start with the expression for a local bulk operator in Rindler coordinates [7],

$$
\begin{aligned}
\Phi^{(0)}(r, \phi, t)= & \frac{(\Delta-1) 2^{\Delta-2}}{\pi r_{+}^{\Delta}} \\
& \times \int d x d y\left(\frac{r}{r_{+}}\left(\cos y-\sqrt{1-\frac{r_{+}^{2}}{r^{2}}} \cosh x\right)\right)^{\Delta-2} \mathcal{O}_{\text {Rindler }}\left(\phi+i \frac{l y}{r_{+}}, t+\frac{l^{2} x}{r_{+}}\right)
\end{aligned}
$$

where the $\mathrm{AdS}_{3}$ metric is

$$
d s^{2}=-\frac{r^{2}-r_{+}^{2}}{l^{2}} d t^{2}+\frac{l^{2}}{r^{2}-r_{+}^{2}} d r^{2}+r^{2} d \phi^{2}
$$

Here $l$ is the AdS radius, $r_{+}$is the horizon radius and the region of integration is

$$
\cos y>\sqrt{1-\frac{r_{+}^{2}}{r^{2}}} \cosh x .
$$

The Rindler operator in the CFT is normalized according to $\lim _{r \rightarrow \infty} r^{\Delta} \Phi^{(0)}(r, \phi, t)=$ $\mathcal{O}_{\text {Rindler }}(\phi, t)$. We understand the analytic continuation to complex boundary coordinates to be defined by

$$
\mathcal{O}_{\text {Rindler }}\left(\phi+i \frac{l y}{r_{+}}, t+\frac{l^{2} x}{r_{+}}\right)=\int d \omega d k e^{-i \omega\left(t+\frac{l^{2} x}{r_{+}}\right)} e^{i k\left(\phi+i \frac{l y}{r_{+}}\right)} \mathcal{O}_{\text {Rindler }}(\omega, k)
$$

As $r \rightarrow r_{+}$the integration region becomes $-\infty<x<\infty$ and $-\frac{\pi}{2}<y<\frac{\pi}{2}$. Thus

$\Phi^{(0)}\left(r_{+}, \phi, t\right)=\frac{(\Delta-1) 2^{\Delta-2}}{\pi r_{+}^{\Delta}} \int_{-\frac{\pi}{2}}^{\frac{\pi}{2}} d y \cos ^{\Delta-2} y \int d \omega d k e^{-i \omega t} e^{i k\left(\phi+i \frac{l y}{r_{+}}\right)} \mathcal{O}_{\operatorname{Rindler}}(\omega, k) \int_{-\infty}^{\infty} d x e^{-i \omega \frac{l^{2} x}{r_{+}}}$ 
The integral over $x$ sets $\omega$ to zero. Then using

$$
\int_{-\frac{\pi}{2}}^{\frac{\pi}{2}} d y \cos ^{\Delta-2} y e^{-k l y / r_{+}}=\frac{\Gamma(\Delta)}{2^{\Delta-1}(\Delta-1)} \frac{1}{\left|\Gamma\left(\frac{\Delta}{2}+\frac{i k l}{2 r_{+}}\right)\right|^{2}}
$$

and defining

$$
\Phi^{(0)}\left(r_{+}, k, t\right)=\frac{1}{2 \pi} \int_{-\infty}^{\infty} d \phi e^{-i k \phi} \Phi^{(0)}(r, \phi, t)
$$

we get

$$
\Phi^{(0)}\left(r_{+}, k, t\right)=\frac{\Gamma(\Delta)}{l r_{+}^{\Delta-1}} \frac{1}{\left|\Gamma\left(\frac{\Delta}{2}+\frac{i k l}{2 r_{+}}\right)\right|^{2}} \mathcal{O}_{\text {Rindler }}(\omega=0, k)
$$

This shows that zero frequency modes relative to the boundary Rindler Hamiltonian live on the bulk RT surface, and due to (2.17), that bulk objects on the RT surface commute with $H_{\text {mod }}$. Setting $k=0$ in $(2.25)$ gives

$$
\frac{1}{2 \pi} \int_{-\infty}^{\infty} d \phi \Phi^{(0)}(r, \phi, t)=\frac{\Gamma(\Delta)}{l r_{+}^{\Delta-1} \Gamma^{2}\left(\frac{\Delta}{2}\right)} \mathcal{O}_{\text {Rindler }}(\omega=0, k=0)
$$

where the left-hand side is up to a constant the integral of the bulk field over the RT surface which serves as the horizon of the bulk Rindler wedge.

In fact (2.26) follows from results in the literature. The integral of a bulk field operator over an RT surface $\gamma$ was identified in [20, 22] with a particular CFT expression. In two dimensions, for a primary operator with dimensions $h=\bar{h}=\frac{1}{2} \Delta_{\mathcal{O}}$, the appropriate identification was found to be

$$
\begin{aligned}
Q(\mathcal{O} ; u, u ; \bar{v}, \bar{v}) & =\frac{C_{\mathrm{blk}}}{8 \pi G_{N}} \int_{\gamma} d s \Phi^{(0)} \\
& =C_{\mathcal{O}} \int_{D(x, y)} d \xi d \bar{\xi}\left(\frac{(u-\xi)(\xi-v)}{(u-v)}\right)^{h-1}\left(\frac{(\bar{v}-\bar{\xi})(\bar{\xi}-\bar{u})}{(\bar{v}-\bar{u})}\right)^{\bar{h}-1} \mathcal{O}(\xi, \bar{\xi})
\end{aligned}
$$

where $C_{\mathrm{blk}}$ and $C_{\mathcal{O}}$ are normalization constants. To see what the right hand side of (2.27) represents, we make a conformal transformation $\xi \rightarrow \eta$ and $\bar{\xi} \rightarrow \bar{\eta}$ as in (2.9) and define a Rindler operator $\mathcal{O}_{R}$ as the conformal transformation ${ }^{5}$ of $\mathcal{O}$

$$
\mathcal{O}_{R}(\phi, \hat{t})=\left(\frac{(u-\xi)(\xi-v)}{(u-v)}\right)^{h}\left(\frac{(\bar{v}-\bar{\xi})(\bar{\xi}-\bar{u})}{(\bar{v}-\bar{u})}\right)^{\bar{h}} \mathcal{O}(\xi, \bar{\xi})
$$

Then we see that

$$
\begin{aligned}
Q(\mathcal{O} ; u, \bar{u} ; v, \bar{v}) & =C_{\mathcal{O}} \int_{-\infty}^{\infty} \int_{-\infty}^{\infty} d \hat{t} d \phi \mathcal{O}_{R}(\hat{t}, \phi) \\
& =C_{\mathcal{O}} \mathcal{O}_{R}(\omega=0, k=0)
\end{aligned}
$$

which up to constants agrees with (2.26).

\footnotetext{
${ }^{5}$ The relation between $\mathcal{O}_{\text {Rindler }}$ and $\mathcal{O}$ is usually taken to be $\mathcal{O}_{\text {Rindler }}=\lim _{r \rightarrow \infty}(r Z)^{\Delta} \mathcal{O}$. See (39) in [8]. This normalization gives an extra factor of $r_{+}^{\Delta}$ compared to $(2.28)$, so that $\mathcal{O}_{\text {Rindler }}=r_{+}^{\Delta} \mathcal{O}_{R}$.
} 
It will be useful below to show directly that $Q$ commutes with $H_{\text {mod }}$. In Lorentzian CFT the commutator of the energy-momentum tensor with a primary field is

$$
\begin{aligned}
& 2 \pi\left[T_{w w}(w), \mathcal{O}\right]=2 \pi i\left(h \partial_{\xi} \delta(\xi-w) \mathcal{O}+\delta(\xi-w) \partial_{\xi} \mathcal{O}\right) \\
& 2 \pi\left[T_{\bar{w} \bar{w}}(\bar{w}), \mathcal{O}\right]=-2 \pi i\left(h \partial_{\bar{\xi}} \delta(\bar{\xi}-\bar{w}) \mathcal{O}+\delta(\bar{\xi}-\bar{w}) \partial_{\bar{\xi}} \mathcal{O}\right)
\end{aligned}
$$

Now we can compute the action of the modular Hamiltonian on a CFT operator. We start with the modular Hamiltonian for a segment $\left(y_{1}, y_{2}\right)$ on the $T=0$ time slice, $H_{\text {mod }}=$ $H_{\text {mod }}^{(R)}+H_{\text {mod }}^{(L)}$ where

$$
\begin{aligned}
& H_{\text {mod }}^{(R)}=2 \pi \int_{y_{1}}^{y_{2}} \frac{\left(w-y_{1}\right)\left(y_{2}-w\right)}{y_{2}-y_{1}} T_{w w}(w) \\
& H_{\text {mod }}^{(L)}=2 \pi \int_{y_{1}}^{y_{2}} \frac{\left(\bar{w}-y_{1}\right)\left(y_{2}-\bar{w}\right)}{y_{2}-y_{1}} T_{\bar{w} \bar{w}}(\bar{w})
\end{aligned}
$$

We find

$$
\begin{aligned}
& {\left[H_{\text {mod }}^{(R)}, \mathcal{O}(\xi, \bar{\xi})\right]=\Theta\left(\left(\xi-y_{1}\right)\left(y_{2}-\xi\right)\right) \frac{2 \pi i}{y_{2}-y_{1}}\left(h\left(y_{2}+y_{1}-2 \xi\right)+\left(\xi-y_{1}\right)\left(y_{2}-\xi\right) \partial_{\xi}\right) \mathcal{O}(\xi, \bar{\xi})} \\
& {\left[H_{\text {mod }}^{(L)}, \mathcal{O}(\xi, \bar{\xi})\right]=-\Theta\left(\left(\bar{\xi}-y_{1}\right)\left(y_{2}-\bar{\xi}\right)\right) \frac{2 \pi i}{y_{2}-y_{1}}\left(h\left(y_{2}+y_{1}-2 \bar{\xi}\right)+\left(\bar{\xi}-y_{1}\right)\left(y_{2}-\bar{\xi}\right) \partial_{\bar{\xi}}\right) \mathcal{O}(\xi, \bar{\xi})}
\end{aligned}
$$

One can then easily check that $Q(\mathcal{O} ; u, u ; \bar{v}, \bar{v})$ commutes with $H_{\bmod }$. In fact $Q$ is the unique expression which commutes with both $H_{\text {mod }}^{(R)}$ and $H_{\text {mod }}^{(L)}$. To see this act on $(2.27)$ using (2.31) and integrate by parts.

In fact there are generalizations of (2.29). A mode of the boundary Rindler operator with zero frequency but non-zero momentum $Q_{k} \equiv \mathcal{O}_{R}(\omega=0, k)$ can be written as

$$
Q_{k}=\int_{D(x, y)} d \xi d \bar{\xi}\left(\frac{(\xi-v)(\bar{\xi}-\bar{u})}{(u-\xi)(\bar{v}-\bar{\xi})}\right)^{\frac{i k}{2}}\left(\frac{(u-\xi)(\xi-v)}{(u-v)}\right)^{h-1}\left(\frac{(\bar{v}-\bar{\xi})(\bar{\xi}-\bar{u})}{(\bar{v}-\bar{u})}\right)^{\bar{h}-1} \mathcal{O}(\xi, \bar{\xi})
$$

This commutes with the sum $H_{\text {mod }}^{(R)}+H_{\text {mod }}^{(L)}$ but obeys $\left[H_{\text {mod }}^{(R)}-H_{\text {mod }}^{(L)}, Q_{k}\right]=2 \pi k Q_{k}$. From $(2.25) Q_{k}$ is related to the integral of $\Phi^{(0)}$ over the RT surface with a particular weight.

\subsection{Extended modular Hamiltonian}

In what follows we will want to compare the action of two modular Hamiltonians based on different segments of the boundary. To make this comparison it is very convenient to define what we call an extended modular Hamiltonian $\tilde{H}_{\text {mod. }}{ }^{6}$ The extended modular Hamiltonian agrees with the usual modular Hamiltonian within its defining segment, but it extends in a natural way to be non-zero outside the segment. Thus the action of $\tilde{H}_{\text {mod }}$ on operators inside the diamond $D(x, y)$ based on the segment will be the same as the

\footnotetext{
${ }^{6}$ This quantity has appeared before in the literature. In $[19,29] \tilde{H}_{\text {mod }}$ was referred to as the total modular operator $K$, and in [30] it was referred to as the full modular operator $\widehat{K}$.
} 
action of the usual modular Hamiltonian, but $H_{\text {mod }}$ and $\tilde{H}_{\text {mod }}$ act differently on operators outside the diamond.

A convenient definition of the extended modular Hamiltonian for a segment $\left(y_{1}, y_{2}\right)$ of the boundary at $T=0$ is just

$$
\begin{aligned}
& \tilde{H}_{\text {mod }}^{(R)}=2 \pi \int_{-\infty}^{\infty} \frac{\left(w-y_{1}\right)\left(y_{2}-w\right)}{y_{2}-y_{1}} T_{w w}(w) \\
& \tilde{H}_{\text {mod }}^{(L)}=2 \pi \int_{-\infty}^{\infty} \frac{\left(\bar{w}-y_{1}\right)\left(y_{2}-\bar{w}\right)}{y_{2}-y_{1}} T_{\bar{w} \bar{w}}(\bar{w})
\end{aligned}
$$

Compared to the usual definition (2.30) all we've done is extend the limits of integration. This definition of the extended modular Hamiltonian has a natural interpretation. As we show in appendix A, $\tilde{H}_{\text {mod }}$ can be identified with the modular Hamiltonian for an interval $A$ on the boundary minus the modular Hamiltonian for its complement $\bar{A}{ }^{7}$

$$
\tilde{H}_{\text {mod }, A}=H_{\text {mod }, A}-H_{\text {mod }, \bar{A}}
$$

This has the nice feature that $\tilde{H}_{\text {mod, } A}$ generates a non-trivial flow everywhere in the bulk, except on the RT surface associated with $A$ which it leaves invariant. This means operators which commute with $\tilde{H}_{\text {mod }}$ must be localized on the RT surface. It follows from the definition that

$$
\begin{aligned}
{\left[\tilde{H}_{\text {mod }}^{(R)}, \mathcal{O}(\xi, \bar{\xi})\right] } & =\frac{2 \pi i}{y_{2}-y_{1}}\left(h\left(y_{2}+y_{1}-2 \xi\right)+\left(\xi-y_{1}\right)\left(y_{2}-\xi\right) \partial_{\xi}\right) \mathcal{O}(\xi, \bar{\xi}) \\
{\left[\tilde{H}_{\text {mod }}^{(L)}, \mathcal{O}(\xi, \bar{\xi})\right] } & =-\frac{2 \pi i}{y_{2}-y_{1}}\left(h\left(y_{2}+y_{1}-2 \bar{\xi}\right)+\left(\bar{\xi}-y_{1}\right)\left(y_{2}-\bar{\xi}\right) \partial_{\bar{\xi}}\right) \mathcal{O}(\xi, \bar{\xi})
\end{aligned}
$$

The action of the extended total modular Hamiltonian $\tilde{H}_{\text {mod }}^{(L)}+\tilde{H}_{\text {mod }}^{(R)}$ on a primary field is

$$
\left[\tilde{H}_{\text {mod }}, \mathcal{O}(\xi, \bar{\xi})\right]=\frac{2 \pi i}{y_{2}-y_{1}}\left((\bar{\xi}-\xi) \Delta-y_{1} y_{2}\left(\partial_{\xi}-\partial_{\bar{\xi}}\right)+\left(y_{1}+y_{2}\right)\left(\xi \partial_{\xi}-\bar{\xi} \partial_{\bar{\xi}}\right)+\bar{\xi}^{2} \partial_{\bar{\xi}}-\xi^{2} \partial_{\xi}\right) \mathcal{O}
$$

Compared to the action of the usual modular Hamiltonian (2.31), the only change is that there are no step functions.

Let us now look at a local bulk operator in the Poincaré patch and show that it commutes with the extended modular Hamiltonian appropriate for a segment whose RT surface passes through the bulk point. The bulk operator in Poincaré coordinates can be written using the complexified smearing function as

$$
\Phi(Z, X, T)=\frac{\Delta-1}{\pi} \int_{y^{\prime 2}+t^{\prime 2}<Z^{2}}\left(\frac{Z^{2}-y^{\prime 2}-t^{\prime 2}}{Z}\right)^{\Delta-2} \mathcal{O}\left(T+t^{\prime}, X+i y^{\prime}\right)
$$

We understand the complexified spatial coordinate as corresponding to the formal expression

$$
\Phi(Z, X, T=0)=\frac{\Delta-1}{\pi} \int_{y^{\prime 2}+t^{\prime 2}<Z^{2}}\left(\frac{Z^{2}-y^{\prime 2}-t^{\prime 2}}{Z}\right)^{\Delta-2} e^{i y^{\prime} \frac{d}{d X}} \mathcal{O}\left(t^{\prime}, X\right)
$$

\footnotetext{
${ }^{7}$ We are grateful to Michal Heller for suggesting this connection.
} 
Then $\left(\xi=X-t^{\prime}, \bar{\xi}=X+t^{\prime}\right)$

$$
\begin{aligned}
{\left[\tilde{H}_{\text {mod }}, \Phi\right]=} & \frac{2 i(\Delta-1)}{y_{2}-y_{1}} \int_{y^{\prime 2}+t^{\prime 2}<Z^{2}}\left(\frac{Z^{2}-y^{\prime 2}-t^{\prime 2}}{Z}\right)^{\Delta-2} e^{i y^{\prime}\left(\frac{d}{d \xi}+\frac{d}{d \bar{\xi}}\right)} \\
& \left((\bar{\xi}-\xi) \Delta-y_{1} y_{2}\left(\partial_{\xi}-\partial_{\bar{\xi}}\right)+\left(y_{1}+y_{2}\right)\left(\xi \partial_{\xi}-\bar{\xi} \partial_{\bar{\xi}}\right)+\bar{\xi}^{2} \partial_{\bar{\xi}}-\xi^{2} \partial_{\xi}\right) \mathcal{O}(\xi, \bar{\xi})
\end{aligned}
$$

We now define $q=\xi+i y^{\prime}$ and $p=\bar{\xi}+i y^{\prime}$, so the above expression is

$$
\begin{aligned}
{\left[\tilde{H}_{\text {mod }}, \Phi\right]=} & \frac{2 i(\Delta-1)}{y_{2}-y_{1}} \int_{Z^{2}+(q-X)(p-X)>0}\left(\frac{Z^{2}+(q-X)(p-X)}{Z}\right)^{\Delta-2} \\
& \left((p-q) \Delta-y_{1} y_{2}\left(\partial_{q}-\partial_{p}\right)+\left(y_{1}+y_{2}\right)\left(q \partial_{q}-p \partial_{p}\right)+p^{2} \partial_{p}-q^{2} \partial_{q}\right) \mathcal{O}(q, p)
\end{aligned}
$$

Now we can integrate by parts and after a little algebra we find that

$$
\left[\tilde{H}_{\text {mod }}, \Phi(Z, X, T=0)\right]=0
$$

provided that

$$
Z^{2}-\left(y_{1}+y_{2}\right) X+y_{1} y_{2}+X^{2}=0
$$

This is simply the condition that the bulk point $(Z, X, T=0)$ lies on a spacelike geodesic whose endpoints hit the boundary at $\left(T=0, y_{1}\right)$ and $\left(T=0, y_{2}\right)$. See $(2.14)$.

\subsection{Finite temperature}

In this section we extend the previous discussion to treat a modular Hamiltonian which is not constructed from the ground state of the CFT. Instead we consider a CFT at finite temperature.

For a CFT on a line at finite temperature $\beta^{-1}=\frac{r_{+}}{2 \pi l^{2}}$ the modular Hamiltonian for a region $(-R, R)$ is given by $[31,32]$

$$
H_{\mathrm{mod}}=c\left(\int_{-R}^{R}\left(\cosh \frac{r_{+} R}{l^{2}}-\cosh \frac{r_{+} \xi}{l^{2}}\right) T_{\xi \xi}(\xi)+\int_{-R}^{R}\left(\cosh \frac{r_{+} R}{l^{2}}-\cosh \frac{r_{+} \bar{\xi}}{l^{2}}\right) T_{\bar{\xi} \bar{\xi}}(\bar{\xi})\right)
$$

with $c=\frac{2 l^{2}}{r_{+}} \sinh \frac{r_{+} R}{l^{2}}$. The extended modular Hamiltonian for the same region is then given by

$$
\tilde{H}_{\mathrm{mod}}=c\left(\int_{-\infty}^{\infty}\left(\cosh \frac{r_{+} R}{l^{2}}-\cosh \frac{r_{+} \xi}{l^{2}}\right) T_{\xi \xi}(\xi)+\int_{-\infty}^{\infty}\left(\cosh \frac{r_{+} R}{l^{2}}-\cosh \frac{r_{+} \bar{\xi}}{l^{2}}\right) T_{\bar{\xi} \bar{\xi}}(\bar{\xi})\right)
$$

The action of the extended Hamiltonian on a primary scalar operator of dimension $2 h$ is

$$
\begin{aligned}
{\left[\tilde{H}_{\text {mod }}, \mathcal{O}\right]=} & c\left[-\frac{r_{+} h}{l^{2}} \sinh \frac{r_{+} \xi}{l^{2}}+\frac{r_{+} h}{l^{2}} \sinh \frac{r_{+} \bar{\xi}}{l^{2}}\right. \\
& \left.+\left(\cosh \frac{r_{+} R}{l^{2}}-\cosh \frac{r_{+} \xi}{l^{2}}\right) \partial_{\xi}-\left(\cosh \frac{r_{+} R}{l^{2}}-\cosh \frac{r_{+} \bar{\xi}}{l^{2}}\right) \partial_{\bar{\xi}}\right] \mathcal{O}(\xi, \bar{\xi})
\end{aligned}
$$


We want to show that this modular Hamiltonian commutes with a local bulk operator on the corresponding RT surface. The bulk operator has a representation using complexified coordinates as in (2.18),

$$
\Phi^{(0)}(r, \phi, t=0) \sim\left(\frac{r}{r_{+}}\right)^{\Delta-2} \int d x d y\left(\cos y-\sqrt{1-\frac{r_{+}^{2}}{r^{2}}} \cosh x\right)^{\Delta-2} \mathcal{O}\left(\phi+\frac{i l y}{r_{+}}, \frac{l^{2} x}{r_{+}}\right)
$$

where both $x, y$ are real and the region of integration is $\cos y>\sqrt{1-\frac{r_{+}^{2}}{r^{2}}} \cosh x$. We understand the operator at complex boundary coordinates to be defined by

$$
\mathcal{O}\left(\phi+\frac{i l y}{r_{+}}, \frac{l^{2} x}{r_{+}}\right)=e^{i \frac{l y}{r_{+}} \frac{d}{d \phi}} \mathcal{O}\left(\phi, \frac{l^{2} x}{r_{+}}\right)
$$

Using (2.45) and (2.48) we get $\left(q=l \phi-\frac{l^{2} x}{r_{+}}+i \frac{l^{2} y}{r_{+}}\right.$and $\left.p=l \phi+\frac{l^{2} x}{r_{+}}+i \frac{l^{2} y}{r_{+}}\right)$

$$
\begin{aligned}
& {\left[\tilde{H}_{\mathrm{mod}}, \Phi^{(0)}(r, \phi, t=0)\right] } \\
\sim & c\left(\frac{r}{r_{+}}\right)^{\Delta-2} \int d x d y\left(\cosh \left(\frac{r_{+}}{l^{2}} \frac{p+q-2 l \phi}{2}\right)-\sqrt{1-\frac{r_{+}^{2}}{r^{2}}} \cosh \left(\frac{r_{+}}{l^{2}} \frac{p-q}{2}\right)\right)^{\Delta-2} \times \\
& \left(\frac{r_{+}}{l^{2}} h\left(\sinh \frac{r_{+}}{l^{2}} p-\sinh \frac{r_{+}}{l^{2}} q\right)+\left(\cosh \frac{r_{+} R}{l^{2}}-\cosh \frac{r_{+} q}{l^{2}}\right) \partial_{q}-\left(\cosh \frac{r_{+} R}{l^{2}}-\cosh \frac{r_{+} p}{l^{2}}\right) \partial_{p}\right) \mathcal{O}(q, p)
\end{aligned}
$$

After integrating by parts and a little algebra one finds that

$$
\left[\tilde{H}_{\text {mod }}, \Phi^{(0)}(r, \phi, t=0)\right]=0
$$

provided

$$
\sqrt{1-\frac{r_{+}^{2}}{r^{2}}}=\frac{\cosh \frac{r_{+}}{l} \phi}{\cosh \frac{r_{+}}{l^{2}} R}
$$

As shown in appendix $\mathrm{C}$, this condition is satisfied provided the bulk point $(r, \phi, t=0)$ lies on a spacelike geodesic connecting the two boundary points $(t=0, l \phi=-R)$ and $(t=0, l \phi=R)$.

\section{Bulk operators from intersecting modular Hamiltonians}

We saw that a bulk operator $\Phi$ living on the RT surface associated with a segment of the boundary commutes with the modular Hamiltonian appropriate to that segment. Of course this does not imply that $\Phi$ is local in the bulk. But if there is another segment on the boundary whose RT surface intersects the RT surface of the first one at a point, then we can demand that $\Phi$ commutes with both modular Hamiltonians. In this case $\Phi$ must be a local bulk operator living on the intersection point.

To make a connection to other work, note that on a formal level the action of the extended modular Hamiltonian appropriate for the vacuum state of a CFT on a CFT primary given in (2.37) identifies it as

$$
\tilde{H}_{\mathrm{mod}}=\frac{2 \pi}{y_{2}-y_{1}}\left(Q_{0}+y_{1} y_{2} P_{0}+\left(y_{1}+y_{2}\right) M_{01}\right)
$$


Here $Q_{0}, P_{0}, M_{01}$ are generators of the conformal group.

$$
Q_{0}=i\left(\bar{L}_{1}-L_{1}\right) \quad P_{0}=i\left(\bar{L}_{-1}-L_{-1}\right), \quad M_{01}=i\left(\bar{L}_{0}-L_{0}\right)
$$

So given two segments of the boundary $\left(y_{1}, y_{2}\right)$ and $\left(y_{3}, y_{4}\right)$, the condition for a bulk operator to live on the intersection of the corresponding RT surface becomes

$$
\left[\left(Q_{0}+y_{1} y_{2} P_{0}+\left(y_{1}+y_{2}\right) M_{01}\right), \Phi\right]=0, \quad\left[\left(Q_{0}+y_{3} y_{4} P_{0}+\left(y_{3}+y_{4}\right) M_{01}\right), \Phi\right]=0
$$

In $[24,26]$, similar conditions were obtained for the special case of a bulk operator in the center of AdS by symmetry considerations.

In this section we will solve (3.3) in coordinate space to recover the smearing function for a local bulk operator in the complex coordinate representation. We will do the same thing in momentum space and recover the bulk Poincaré modes which make up a local bulk operator. In addition we will solve the appropriate equations for a CFT at finite temperature and recover a local bulk operator in the BTZ background. This provides a new way of constructing the zeroth-order bulk operator and deriving bulk modes without knowing anything about the bulk geometry.

Note however that the conditions for bulk locality (3.3) only determine the bulk operator up to a coefficient. The coefficient could depend on bulk position, so in fact we can only generically recover $\Phi^{(0)}$ up to a function of the bulk space-time coordinates. In states where the CFT has an unbroken spacetime translation symmetry the function can only depend on the bulk radial coordinate. In this case dimensional analysis fixes $\Phi^{(0)}$ up to an overall constant. But in general locality is not enough to fix the function. Even with this freedom we get quite a lot of information. For example, given the two-point function of a local bulk operator with another local bulk or boundary operator we can identify the singularities and deduce the bulk causal structure. ${ }^{8}$ Also the program of perturbatively correcting the zeroth-order bulk operator to take interactions into account only relies on the singularity structure, so up to a multiplicative coefficient an interacting local bulk operator could be constructed. Moreover this multiplicative freedom cancels in any ratio of correlation functions involving a fixed bulk operator with any number of boundary operators, so one could determine these ratios unambiguously. As another example of an unambiguous quantity, along the way we will see that the construction generates the equations which describe bulk spacelike geodesics.

\subsection{Recovering smearing functions for the vacuum state}

We start with an ansatz for an object that commutes with the modular Hamiltonian

$$
\Phi(X)=\int d t^{\prime} d y^{\prime} g(p, q) \mathcal{O}(q, p)
$$

where $q=X-t^{\prime}+i y^{\prime}, p=X+t^{\prime}+i y^{\prime}$. In the ansatz $t^{\prime}$ and $y^{\prime}$ are taken to be real and $X$ is left as a free real variable. From (2.41) the action of the modular Hamiltonian for a

\footnotetext{
${ }^{8}$ One can use this information to reconstruct the bulk metric, up to a conformal factor, by the method of light-cone cuts [33].
} 
segment $\left(y_{1}, y_{2}\right)$ on $\Phi$ is given by

$$
\begin{aligned}
{\left[\tilde{H}_{\text {mod }}^{12}, \Phi(X)\right]=} & \frac{2 \pi i}{y_{2}-y_{1}} \int d t^{\prime} d y^{\prime} g(p, q) \times \\
& \left((p-q) \Delta-y_{1} y_{2}\left(\partial_{q}-\partial_{p}\right)+\left(y_{1}+y_{2}\right)\left(q \partial_{q}-p \partial_{p}\right)+p^{2} \partial_{p}-q^{2} \partial_{q}\right) \mathcal{O}(q, p)
\end{aligned}
$$

We take two such modular Hamiltonians with parameters $\left(y_{1}, y_{2}\right)$ and $\left(y_{3}, y_{4}\right)$ and demand

$$
\left(y_{2}-y_{1}\right)\left[\tilde{H}_{\mathrm{mod}}^{12}, \Phi(\xi, \bar{\xi})\right]=0, \quad\left(y_{4}-y_{3}\right)\left[\tilde{H}_{\mathrm{mod}}^{34}, \Phi(\xi, \bar{\xi})\right]=0
$$

It's convenient to first look at the difference of the equations in (3.6),

$$
\int d t^{\prime} d y^{\prime} g(p, q)\left(\left(y_{3} y_{4}-y_{1} y_{2}\right)\left(\partial_{q}-\partial_{p}\right)+\left(y_{1}+y_{2}-y_{3}-y_{4}\right)\left(q \partial_{q}-p \partial_{p}\right)\right) \mathcal{O}(q, p)=0
$$

After integration by parts this gives an equation for $g(q, p)$,

$$
\left(\left(X_{0}-q\right) \partial_{q}-\left(X_{0}-p\right) \partial_{p}\right) g(q, p)=0
$$

where $X_{0}=\frac{y_{1} y_{2}-y_{3} y_{4}}{y_{1}+y_{2}-y_{3}-y_{4}}$. The solution to this equation is

$$
g(q, p)=f\left(\left(p-X_{0}\right)\left(q-X_{0}\right)\right)
$$

where $f$ is an arbitrary function. We now use this form and solve the equation $\left[\tilde{H}_{\text {mod }}^{12}, \Phi(X)\right]=0$. Following the same steps as before we get an equation for $f$

$$
\left((\Delta-2)(p-q)+y_{1} y_{2}\left(\partial_{q}-\partial_{p}\right)-\left(y_{1}+y_{2}\right)\left(q \partial_{q}-p \partial_{p}\right)+q^{2} \partial_{q}-p^{2} \partial_{p}\right) f\left(\left(q-X_{0}\right)\left(p-X_{0}\right)\right)=0
$$

whose solution is

$$
f=c_{\Delta}\left(Z^{2}+\left(p-X_{0}\right)\left(q-X_{0}\right)\right)^{\Delta-2}
$$

where

$$
Z^{2}=\left(y_{1}+y_{2}\right) X_{0}-y_{1} y_{2}-X_{0}^{2}
$$

The two parameters appearing in the solution $X_{0}, Z$ can be identified as the coordinates of the local operator in the bulk. Note, for example, that as $y_{1}, y_{3} \rightarrow y_{2}$ we have $Z \rightarrow 0$ and $X_{0} \rightarrow y_{2}$. Comparing (3.12) to (2.14), note that we have recovered from the CFT the equation which describes a spacelike geodesic in the bulk.

For the integration by parts to work without any boundary terms we need the integration region to be bounded by $Z^{2}+\left(p-X_{0}\right)\left(q-X_{0}\right)=0$. For this to be possible for real $\left(t^{\prime}, y^{\prime}\right)$ we see that we must have $X=X_{0}$. So finally we get

$$
\Phi\left(Z, X_{0}\right)=c_{\Delta} \int_{t^{\prime 2}+y^{\prime 2}<Z^{2}} d t^{\prime} d y^{\prime}\left(Z^{2}-t^{\prime 2}-y^{\prime 2}\right)^{\Delta-2} \mathcal{O}\left(t^{\prime}, X_{0}+i y^{\prime}\right) .
$$

Since the vacuum state is translation invariant we expect correlation functions of local bulk fields to be translation invariant as well. From this we can deduce that the coefficient $c_{\Delta}$ is a function of $Z$ only, which could be determined from a normalization condition such as $\Phi(Z \rightarrow 0, X) \rightarrow \frac{Z^{\Delta}}{2 \Delta-d} \mathcal{O}(X)$. In this way we have recovered the bulk operator written in the complex coordinate representation. 


\subsubsection{Derivative representation}

Another possible representation for a bulk operator is

$$
\Phi(Z, \xi, \bar{\xi})=\sum_{n, m=0}^{\infty} a_{n m} \partial_{\xi}^{n} \partial_{\bar{\xi}}^{m} \mathcal{O}(\xi, \bar{\xi})
$$

In this case formally we can impose locality using the usual modular Hamiltonian and we do not need the extended modular Hamiltonian.

We wish to impose the conditions (3.6), that $\Phi$ commutes with two modular Hamiltonians. As before we start by looking at the difference of the two equations in (3.6) which gives

$$
\sum_{n, m=0}^{\infty} a_{n m} \partial_{\xi}^{n} \partial_{\bar{\xi}}^{m}\left(\left(\xi-X_{0}\right) \partial_{\xi}+\left(X_{0}-\bar{\xi}\right) \partial_{\bar{\xi}}\right) \mathcal{O}(\xi, \bar{\xi})
$$

Without loss of generality we take

$$
\Phi\left(X_{0}\right)=\sum_{n, m=0}^{\infty} a_{n m} \partial_{\xi}^{n} \partial_{\bar{\xi}}^{m} \mathcal{O}(\xi, \bar{\xi})_{\xi=\bar{\xi}=X_{0}}
$$

Using this in (3.15) and setting the coefficients of $\partial_{\xi}^{n} \partial_{\bar{\xi}}^{m} \mathcal{O}\left(\xi=X_{0}, \bar{\xi}=X_{0}\right)$ to zero gives

$$
(n-m) a_{n m}=0
$$

So in fact $\Phi$ must have the form

$$
\Phi\left(X_{0}\right)=\sum_{m=0}^{\infty} a_{m}\left(\partial_{\xi} \partial_{\bar{\xi}}\right)^{m} \mathcal{O}(\xi, \bar{\xi})_{\xi=\bar{\xi}=X_{0}}
$$

Now demanding that

$$
\left[\tilde{H}_{\mathrm{mod}}^{12}, \Phi\left(X_{0}\right)\right]=0
$$

gives the condition

$$
\begin{aligned}
\sum_{n} a_{m}(m(\Delta+ & m-1) \partial_{\xi}^{m} \partial_{\bar{\xi}}^{m-1}-m(\Delta+m-1) \partial_{\xi}^{m-1} \partial_{\bar{\xi}}^{m} \\
& \left.+Z^{2} \partial_{\xi}^{m+1} \partial_{\bar{\xi}}^{m}-Z^{2} \partial_{\xi}^{m} \partial_{\bar{\xi}}^{m+1}\right) \mathcal{O}(\xi, \bar{\xi})_{\xi=\bar{\xi}=X_{0}}=0
\end{aligned}
$$

where $Z^{2}=-y_{1} y_{2}+\left(y_{1}+y_{2}\right) X_{0}-X_{0}^{2}$. This implies the recursion relation

$$
a_{m}=-\frac{Z^{2}}{m(\Delta+m-1)} a_{m-1}
$$

whose solution is

$$
a_{m}=a_{0} \frac{(-1)^{m} Z^{2 m}}{\Gamma(m+1) \Gamma(\Delta+m)}
$$

As before time and space translation invariance restrict $a_{0}$ to be a function of $Z$. The expression for a bulk operator in Poincaré coordinates (2.38) can be expanded in derivatives [21].

$$
\Phi(Z, X, T)=\Gamma(\Delta) Z^{\Delta} \sum_{m=0}^{\infty} \frac{(-1)^{m} Z^{2 m}}{\Gamma(m+1) \Gamma(\nu+m+1)}\left(\partial_{\xi} \partial_{\bar{\xi}}\right)^{m} \mathcal{O}(\xi, \bar{\xi})
$$

Comparing this to (3.18) and (3.22) we see that we have recovered the local bulk operator $\Phi(Z, X, T=0)$. 


\section{$3.2 \quad$ Recovering bulk modes}

In this section we wish to recover the momentum space representation for a bulk operator, i.e. the bulk modes. We start with the extended modular Hamiltonian for the segment $\left(y_{1}, y_{2}\right)$

$$
\left[\tilde{H}_{\text {mod }}, \mathcal{O}(\xi, \bar{\xi})\right]=\frac{2 \pi i}{y_{2}-y_{1}}\left((\bar{\xi}-\xi) \Delta-y_{1} y_{2}\left(\partial_{\xi}-\partial_{\bar{\xi}}\right)+\left(y_{1}+y_{2}\right)\left(\xi \partial_{\xi}-\bar{\xi} \partial_{\bar{\xi}}\right)+\bar{\xi}^{2} \partial_{\bar{\xi}}-\xi^{2} \partial_{\xi}\right) \mathcal{O}
$$

We define $k_{+}=\frac{k+\omega}{2}, k_{-}=\frac{\omega-k}{2}$ and

$$
\mathcal{O}\left(k_{+}, k_{-}\right)=\frac{1}{4 \pi^{2}} \int d \xi d \bar{\xi} e^{-i k_{+} \xi+i k_{-} \bar{\xi}} \mathcal{O}(\xi, \bar{\xi})
$$

Using (3.24) one finds

$$
\begin{aligned}
{\left[\tilde{H}_{\text {mod }}, \mathcal{O}\left(k_{+}, k_{-}\right)\right]=} & \frac{2 \pi i}{y_{2}-y_{1}}\left(\left(y_{1}+y_{2}\right)\left(\frac{d}{d k_{-}} k_{-}-\frac{d}{d k_{+}} k_{+}\right)-2 i h\left(\frac{d}{d k_{+}}+\frac{d}{d k_{-}}\right)\right. \\
& \left.+\frac{d^{2}}{d^{2} k_{+}} i k_{+}-\frac{d^{2}}{d^{2} k_{-}} i k_{-}-y_{1} y_{2}\left(i k_{+}+i k_{-}\right)\right) \mathcal{O}\left(k_{+}, k_{-}\right)
\end{aligned}
$$

We now look for operators $\Phi$ which commute with the extended modular Hamiltonians for two segments $\left(y_{1}, y_{2}\right)$ and $\left(y_{3}, y_{4}\right)$.

$$
\left(y_{2}-y_{1}\right)\left[\tilde{H}_{\bmod }^{12}, \Phi\right]=0, \quad\left(y_{4}-y_{3}\right)\left[\tilde{H}_{\bmod }^{34}, \Phi\right]=0
$$

We make the ansatz

$$
\Phi=\int d k_{+} d k_{-} g\left(k_{+}, k_{-}\right) \mathcal{O}\left(k_{+}, k_{-}\right)
$$

We first require that $\Phi$ satisfy the difference of the two equations in (3.27). This gives

$$
\begin{aligned}
& \int d k_{+} d k_{-} g\left(k_{+}, k_{-}\right)(\left(y_{1}+y_{2}-y_{3}-y_{4}\right)\left(\frac{d}{d k_{-}} k_{-}-\frac{d}{d k_{+}} k_{+}\right) \\
&\left.-\left(y_{1} y_{2}-y_{3} y_{4}\right)\left(i k_{+}+i k_{-}\right)\right) \mathcal{O}\left(k_{+}, k_{-}\right)=0
\end{aligned}
$$

Upon integration by parts we get an equation for $g\left(k_{+}, k_{-}\right)$,

$$
\left(k_{+} \frac{d}{d k_{+}}-k_{-} \frac{d}{d k_{-}}\right) g\left(k_{+}, k_{-}\right)=i X_{0}\left(k_{+}+k_{-}\right) g\left(k_{+}, k_{-}\right)
$$

where $X_{0}=\frac{y_{1} y_{2}-y_{3} y_{4}}{y_{1}+y_{2}-y_{3}-y_{4}}$. The general solution to this equation is

$$
g\left(k_{+}, k_{-}\right)=f\left(k_{+} k_{-}\right) e^{i\left(k_{+}-k_{-}\right) X_{0}}
$$

where $f$ is an arbitrary function of $k_{+} k_{-}$.

Having imposed that $\Phi$ commutes with the difference $\left(y_{2}-y_{1}\right) \tilde{H}_{\bmod }^{12}-\left(y_{4}-y_{3}\right) \tilde{H}_{\text {mod }}^{34}$, we now require that $\Phi$ commute with $\tilde{H}_{\text {mod }}^{12}$ itself. We start with the ansatz

$$
\Phi\left(X_{0}\right)=\int d k_{+} d k_{-} f\left(k_{+} k_{-}\right) e^{i\left(k_{+}-k_{-}\right) X_{0}} \mathcal{O}\left(k_{+}, k_{-}\right)
$$


The first equation in (3.27) becomes a condition on $f$. After integration by parts and some algebra we find $\left(x=k_{+} k_{-}=\frac{1}{4}\left(\omega^{2}-k^{2}\right)\right)$

$$
x \frac{d^{2} f}{d^{2} x}+2 h \frac{d f}{d x}+\left(\left(y_{1}+y_{2}\right) X_{0}-y_{1} y_{2}-X_{0}^{2}\right) f=0
$$

The solution is

$$
f(\omega, k)=c_{0}\left(Z^{2}\left(\omega^{2}-k^{2}\right)\right)^{-\nu / 2} J_{\nu}\left(Z \sqrt{\omega^{2}-k^{2}}\right)
$$

with $\Delta=\nu+1$ and $Z^{2}=\left(y_{1}+y_{2}\right) X_{0}-y_{1} y_{2}-X_{0}^{2}$. Time and space translation invariance restrict $c_{0}$ to be a function of $Z$. Then (3.32) becomes $^{9}$

$$
\Phi\left(X_{0}\right)=c_{0} \int d \omega d k e^{i k X_{0}}\left(Z^{2}\left(\omega^{2}-k^{2}\right)\right)^{-\nu / 2} J_{\nu}\left(Z \sqrt{\omega^{2}-k^{2}}\right) \mathcal{O}\left(k_{+}, k_{-}\right)
$$

which is the bulk operator $\Phi\left(Z, X_{0}, T=0\right)$ given in (1.3).

If we had imposed the second equation in (3.27) we would have gotten the same result with $Z^{2}=\left(X_{0}-y_{3}\right)\left(y_{4}-X_{0}\right)$. But in fact these two expressions for $Z$ are the same. As long as the parameters $\left(y_{1}, y_{2}, y_{3}, y_{4}\right)$ assign a real value to $Z$ we have a solution where the point $\left(T=0, Z, X_{0}\right)$ is the bulk point located at the intersection of the two boundary-anchored geodesics.

\subsection{Time dependence}

For completeness we show that the construction of local bulk operators based on intersecting modular Hamiltonians also captures the correct time dependence of bulk fields.

To do this we look at the modular Hamiltonian for a diamond that is shifted in time by an amount $T$. In light-front coordinates (2.4) such a diamond is characterized by

$$
u=y_{2}-T, \bar{u}=y_{1}+T, v=y_{1}-T, \bar{v}=y_{2}+T
$$

The extended modular Hamiltonian acting in momentum space is given by

$$
\begin{aligned}
{\left[\tilde{H}_{\text {mod }}^{L}(T), \mathcal{O}\left(k_{+}, k_{-}\right)\right]=} & \frac{1}{y_{2}-y_{1}}\left(\left(y_{1}+y_{2}-2 T\right)\left(h-\frac{d}{d k_{+}} k_{+}\right)\right. \\
& \left.-2 i h \frac{d}{d k_{+}}+\left(\frac{d^{2}}{d^{2} k_{+}}-\left(y_{1} y_{2}-T\left(y_{1}+y_{2}\right)+T^{2}\right)\right) i k_{+}\right) \mathcal{O}\left(k_{+}, k_{-}\right) \\
{\left[\tilde{H}_{\text {mod }}^{R}(T), \mathcal{O}\left(k_{+}, k_{-}\right)\right]=} & \frac{1}{y_{2}-y_{1}}\left(-\left(y_{1}+y_{2}+2 T\right)\left(h-\frac{d}{d k_{-}} k_{-}\right)\right. \\
& \left.-2 i h \frac{d}{d k_{-}}+\left(\frac{d^{2}}{d^{2} k_{-}}-\left(y_{1} y_{2}+T\left(y_{1}+y_{2}\right)+T^{2}\right)\right) i k_{-}\right) \mathcal{O}\left(k_{+}, k_{-}\right)
\end{aligned}
$$

We look for operators that commute with $\tilde{H}_{\text {mod }}^{\text {total }}=\tilde{H}_{\text {mod }}^{L}+\tilde{H}_{\text {mod }}^{R}$. We make an ansatz

$$
\Phi(X)=\int d k_{+} d k_{-} g\left(k_{+}, k_{-}\right) \mathcal{O}\left(k_{+}, k_{-}\right)
$$

\footnotetext{
${ }^{9}$ We could choose the coefficient $c_{0}$ so that $\Phi$ has the right limit as $Z \rightarrow 0$. Also note that the boundary operator only has modes with $|\omega|>|k|$.
} 
If we take two different boundary segments $\left(y_{1}, y_{2}\right)$ and $\left(y_{3}, y_{4}\right)$ at time $T$ then the conditions we wish to impose are

$$
\left(y_{2}-y_{1}\right)\left[\tilde{H}_{\mathrm{mod}}^{12}, \Phi\right]=0, \quad\left(y_{4}-y_{3}\right)\left[\tilde{H}_{\mathrm{mod}}^{34}, \Phi\right]=0
$$

Taking the difference results in an equation for $g\left(k_{+}, k_{-}\right)$,

$$
\left(k_{+} \frac{d}{d k_{+}}-k_{-} \frac{d}{d k_{-}}\right) g\left(k_{+}, k_{-}\right)=\left(\left(X_{0}-T\right) i k_{+}+\left(X_{0}+T\right) i k_{-}\right) g\left(k_{+}, k_{-}\right)
$$

with $X_{0}=\frac{y_{1} y_{2}-y_{3} y_{4}}{y_{1}+y+2-y_{3}-y_{4}}$. The solution to this equation is

$$
g\left(k_{+}, k_{-}\right)=f\left(k_{+} k_{-}\right) e^{i\left(k_{+}-k_{-}\right) X} e^{-i\left(k_{+}+k_{-}\right) T}
$$

with $f$ an arbitrary function of $k_{+} k_{-}$. Thus our ansatz is now

$$
\Phi\left(X_{0}, T\right)=\int d k_{+} d k_{-} f\left(k_{+} k_{-}\right) e^{i\left(k_{+}-k_{-}\right) X} e^{-i\left(k_{+}+k_{-}\right) T} \mathcal{O}\left(k_{+}, k_{-}\right)
$$

Having imposed the difference, the remaining condition

$$
\left[\tilde{H}_{\bmod }^{12}(T), \Phi\left(X_{0}, T\right)\right]=0
$$

is solved (after some algebra) by

$$
f\left(k_{+} k_{-}\right) \sim\left(Z^{2}\left(\omega^{2}-k^{2}\right)\right)^{-\frac{\Delta-1}{2}} J_{\Delta-1}\left(Z \sqrt{\omega^{2}-k^{2}}\right)
$$

where

$$
Z^{2}=\left(y_{1}+y_{2}\right) X_{0}-y_{1} y_{2}-X_{0}^{2}
$$

Thus we've recovered the full Poincaré bulk mode, including its time dependence, purely from CFT considerations. This shows that we can get the complete zeroth-order expression for a local bulk field using intersecting modular Hamiltonians.

\subsection{Recovering BTZ bulk operators}

In this section we follow the same procedure to construct local bulk scalar fields in a BTZ background. We start with the extended modular Hamiltonian appropriate to a $(1+1)$ dimensional CFT at finite temperature. The extended modular Hamiltonian for a segment has two parameters, the size of the segment $2 L$ and the position of the center of the segment $\phi_{0}$.

$$
\begin{aligned}
\tilde{H}_{\text {mod }, L, \phi_{0}} & =c \int_{-\infty}^{\infty}\left(\cosh \frac{r_{+} L}{l^{2}}-\cosh \frac{r_{+}\left(\xi-\phi_{0}\right)}{l^{2}}\right) T_{\xi \xi}(\xi) \\
& +c \int_{-\infty}^{\infty}\left(\cosh \frac{r_{+} L}{l^{2}}-\cosh \frac{r_{+}\left(\bar{\xi}-\phi_{0}\right)}{l^{2}}\right) T_{\bar{\xi} \bar{\xi}}(\bar{\xi})
\end{aligned}
$$

We will consider two extended Hamiltonians

$$
\tilde{H}_{\bmod , L, \phi_{0}} \quad \text { and } \quad \tilde{H}_{\bmod , R, \phi_{0}=0}
$$


and wish to find a CFT operator $\Phi$ that satisfies

$$
\left[\tilde{H}_{\bmod , L, \phi_{0}}, \Phi\right]=0, \quad\left[\tilde{H}_{\bmod , R, \phi_{0}=0}, \Phi\right]=0
$$

The action of the extended Hamiltonian on a primary scalar operator of dimension $2 h$ is

$$
\begin{aligned}
{\left[\tilde{H}_{\text {mod }, L, \phi_{0}}, \mathcal{O}\right]=} & c\left(-\frac{r_{+} h}{l^{2}} \sinh \frac{r_{+}\left(\xi-\phi_{0}\right)}{l^{2}}+\frac{r_{+} h}{l^{2}} \sinh \frac{r_{+}\left(\bar{\xi}-\phi_{0}\right)}{l^{2}}\right. \\
& +\left(\cosh \frac{r_{+} L}{l^{2}}-\cosh \frac{r_{+}\left(\xi-\phi_{0}\right)}{l^{2}}\right) \partial_{\xi} \\
& \left.-\left(\cosh \frac{r_{+} L}{l^{2}}-\cosh \frac{r_{+}\left(\bar{\xi}-\phi_{0}\right)}{l^{2}}\right) \partial_{\bar{\xi}}\right) \mathcal{O}(\xi, \bar{\xi})
\end{aligned}
$$

We define the variables as before

$$
q=l \phi-\frac{l^{2} x}{r_{+}}+i \frac{l^{2} y}{r_{+}}, \quad p=l \phi+\frac{l^{2} x}{r_{+}}+i \frac{l^{2} y}{r_{+}}
$$

where $\phi$ is a free parameter, and we define rescaled variables

$$
\tilde{q}=\frac{r_{+}}{l^{2}} q, \quad \tilde{p}=\frac{r_{+}}{l^{2}} p, \quad \tilde{L}=\frac{r_{+}}{l^{2}} L, \quad \tilde{\phi}_{0}=\frac{r_{+}}{l} \phi_{0}
$$

Starting with the general ansatz

$$
\Phi=\int d \tilde{p} d \tilde{q} g(\tilde{p}, \tilde{q}) \mathcal{O}(\tilde{p}, \tilde{q})
$$

the condition

$$
\left[\tilde{H}_{\bmod , L, \phi_{0}}, \Phi\right]=0
$$

becomes upon integration by parts ${ }^{10}$

$$
\begin{aligned}
& \left(\left(\cosh \tilde{L}-\cosh \left(\tilde{q}-\tilde{\phi}_{0}\right)\right) \partial_{\tilde{q}}-\left(\cosh \tilde{L}-\cosh \left(\tilde{p}-\tilde{\phi}_{0}\right)\right) \partial_{\tilde{p}}\right) g(\tilde{p}, \tilde{q}) \\
= & (h-1)\left(\sinh \left(\tilde{p}-\tilde{\phi}_{0}\right)-\sinh \left(\tilde{q}-\tilde{\phi}_{0}\right)\right) g(\tilde{p}, \tilde{q})
\end{aligned}
$$

We first impose the condition $\left[\tilde{H}_{\bmod , R, \phi_{0}=0}, \Phi\right]=0$. To do this we set $\tilde{\phi}_{0}=0$ and $\tilde{L}=\tilde{R}$ in (3.55). Then using the method of characteristics, the most general solution to $(3.55)$ is

$$
\begin{aligned}
g(\tilde{p}, \tilde{q}) & =c_{0} f(x) K^{h-1} \\
x & =\frac{\sinh \left(\frac{\tilde{R}+\tilde{q}}{2}\right) \sinh \left(\frac{\tilde{R}+\tilde{p}}{2}\right)}{\sinh \left(\frac{\tilde{R}-\tilde{q}}{2}\right) \sinh \left(\frac{\tilde{R}-\tilde{p}}{2}\right)} \\
K & =\sinh \left(\frac{\tilde{R}+\tilde{q}}{2}\right) \sinh \left(\frac{\tilde{R}+\tilde{p}}{2}\right) \sinh \left(\frac{\tilde{R}-\tilde{q}}{2}\right) \sinh \left(\frac{\tilde{R}-\tilde{p}}{2}\right)
\end{aligned}
$$

\footnotetext{
${ }^{10}$ We will choose the region of integration to ensure that there are no boundary terms.
} 
where $f$ is an arbitrary function and $c_{0}$ is a constant. Since we also want $\Phi$ to obey

$$
\left[\tilde{H}_{\bmod , L, \phi_{0}}, \Phi\right]=0
$$

we re-insert the solution (3.56) into (3.55). This now gives an equation for $f(x)$. After some algebra the equation can be recast as

$$
\begin{aligned}
\frac{d f}{d x} & =\frac{h-1}{x} \frac{x-\alpha}{x+\alpha} f \\
\alpha & =\frac{\sinh \tilde{\phi}_{0} \sinh \tilde{R}+\cosh \tilde{\phi_{0}} \cosh \tilde{R}-\cosh \tilde{L}}{\sinh \tilde{\phi_{0}} \sinh \tilde{R}-\cosh \tilde{\phi_{0}} \cosh \tilde{R}+\cosh \tilde{L}}
\end{aligned}
$$

with solution

$$
f(x)=c_{1}\left(\frac{(x+\alpha)^{2}}{x}\right)^{h-1}
$$

The parameter $\alpha$ can be seen to depend on only two parameters by defining

$$
\tanh \tilde{\phi}_{*}=\frac{1}{\sinh \tilde{\phi}_{0}}\left(\cosh \tilde{\phi}_{0}-\frac{\cosh \tilde{L}}{\cosh \tilde{R}}\right)
$$

and noting that

$$
\alpha=\frac{\cosh \tilde{\phi}_{*} \sinh \tilde{R}+\sinh \tilde{\phi}_{*} \cosh \tilde{R}}{\cosh \tilde{\phi}_{*} \sinh \tilde{R}-\sinh \tilde{\phi}_{*} \cosh \tilde{R}}
$$

We can set the free parameter $\phi$ in (3.51) to be $\phi=\phi_{*}$ so that $g(\tilde{p}, \tilde{q})$ becomes

$$
g(\tilde{p}, \tilde{q})=c_{2}\left(\cosh \left(\frac{\tilde{p}+\tilde{q}}{2}-\tilde{\phi}_{*}\right)-\frac{\cosh \tilde{\phi_{*}}}{\cosh \tilde{R}} \cosh \left(\frac{\tilde{p}-\tilde{q}}{2}\right)\right)^{\Delta-2}=c_{2}\left(\cos y-\frac{\cosh \tilde{\phi}_{*}}{\cosh \tilde{R}} \cosh x\right)^{\Delta-2}
$$

The two parameters of the solution $\tilde{\phi}_{*}$ and $\frac{\cosh \tilde{\phi}_{*}}{\cosh \tilde{R}} \operatorname{can}$ be identified as the coordinate parallel to the boundary and the radial coordinate, respectively, by looking at the limit $\tilde{L} \rightarrow 0$, $\tilde{\phi_{0}} \rightarrow \tilde{R}$. In this limit $\tilde{\phi}_{*} \rightarrow \tilde{R}$ and $\frac{\cosh \tilde{\phi_{*}}}{\cosh \tilde{R}} \rightarrow 1$ as expected.

The region of integration is fixed by requiring that there are no boundary terms when we integrate by parts. This determines the region of integration to be

$$
\cos y>\frac{\cosh \tilde{\phi}_{*}}{\cosh \tilde{R}} \cosh x
$$

On an equal-time geodesic stretching from $-\tilde{R}$ to $\tilde{R}$, we show in appendix $\mathrm{C}$ that the boundary coordinate $\phi$ and the bulk coordinate $r$ are related by

$$
\sqrt{1-\frac{r_{+}^{2}}{r^{2}}}=\frac{\cosh \tilde{\phi}}{\cosh \tilde{R}}
$$

Thus (3.62) is the smearing function for a bulk scalar operator $\Phi\left(r, \phi_{*}, t=0\right)$ in a BTZ background [7]. In appendix $\mathrm{C}$ we show that $\phi_{*}$ in (3.60) is just the $\phi$ coordinate where the bulk geodesics intersect. Thus again we have recovered the bulk space-like geodesics from the CFT. 


\section{Conclusions}

In this paper we have shown that CFT operators which mimic local bulk operators commute with the modular Hamiltonian appropriate for a boundary segment whose RT surface passes through the bulk point. If two RT surfaces intersect at a point in the bulk then a bulk observable localized on the intersection must commute with both modular Hamiltonians. Turning this around, we used this as a new way to construct local bulk observables in the CFT, by constructing CFT quantities which commute with intersecting extended modular Hamiltonians. Along the way we recovered bulk space-like geodesics from the CFT.

The computations done in this paper were for $\mathrm{AdS}_{3} / \mathrm{CFT}_{2}$, but the generalization to higher dimensions is clear. The only complication is that $\mathrm{AdS}_{D}$ requires $D-1$ intersecting RT surfaces to define a bulk point.

It seems clear that at least in principle the construction can be carried out for CFT states which are not the vacuum. Indeed in this paper the finite temperature case was treated successfully. Explicit expressions may be difficult to obtain since we have little control over the modular Hamiltonian for non-vacuum states. Moreover in general the modular Hamiltonian will be non-local, so (unlike the examples treated in this paper) for generic states the approach will not lead to a system of local differential equations for the smearing functions. But in principle the same logic applies and should determine local bulk operators in the appropriately-deformed bulk background geometry.

Another, perhaps related, generalization of the construction in this paper would be to include interactions and make contact with the perturbative procedure developed in [1012]. It would also be interesting to understand if there is a connection to the ideas proposed in $[34,35]$.

In this paper we only considered scalar operators. It would be interesting to extend the construction to bulk fields with spin. For massive vector fields this seems straightforward. Bulk fields with gauge redundancy pose an additional challenge, since due to constraints they aren't local objects in the bulk even at the free field level. ${ }^{11}$ Moreover even for bulk scalars gravitational dressing arises as an interaction effect, and once this is taken into account one cannot localize bulk scalar observables to the intersection of RT surfaces. This means that for free bulk gauge fields, and for interacting bulk scalars, one cannot simply demand that bulk observables commute with intersecting modular Hamiltonians. Whether there is an extension of the approach to deal with these issues is an interesting and important question.

The construction developed in this paper raises more speculative issues as well. For example it seems clear that the construction puts constraints on CFT states which are dual to classical bulk geometries. This comes about because a classical bulk geometry requires that an infinite family of equations, stating that different modular Hamiltonians commute with a smeared CFT operator, must all have a common solution. This restricts the form of the modular Hamiltonians and hence presumably the CFT states that can be dual to classical geometries. It would be interesting to make these restrictions more precise.

\footnotetext{
${ }^{11}$ For a recent treatment of observables for gauge fields see [36].
} 


\section{Acknowledgments}

We are grateful to Michal Heller for valuable comments. The work of DK is supported by U.S. National Science Foundation grant PHY-1519705. The work of GL is supported in part by the Israel Science Foundation under grant 504/13.

\section{A Extended modular Hamiltonian}

Here we show that given an interval $A$ and its complement $\bar{A}$, the extended modular Hamiltonian for the region $A$ can be identified as

$$
\tilde{H}_{\bmod , A}=H_{\bmod , A}-H_{\bmod , \bar{A}}
$$

This generalizes the usual extension of a Rindler time translation outside the Rindler wedge and is analogous to defining a thermofield Hamiltonian.

It's straightforward to show (A.1) for the vacuum state of a CFT on a line. In [32] the modular Hamiltonian for a region was constructed using a conformal transformation with an analogy to electrostatics. Given the appropriate conformal transformation $f(z)$ the modular Hamiltonian for a region $A$ was given by

$$
H_{\bmod }^{(R)}=2 \pi \int_{A} \frac{T_{z z}(z)}{f^{\prime}(z)} d z
$$

and similar for $H_{\bmod }^{(L)}$. If we take the region $B$ to be the union of the segments $\left(-\infty, y_{1}\right)$ and $\left(y_{2}, \infty\right)$, then the appropriate $f(z)$ is just $f(z)=\ln \left(\frac{y_{2}-z}{z-y_{1}}\right)$ and similar for $\bar{f}(\bar{z}){ }^{12}$ This gives the right-moving part of the modular Hamiltonian for this region to be

$$
H_{\mathrm{mod}, B}^{(R)}=-2 \pi \int_{-\infty}^{y_{1}} \frac{\left(y_{2}-z\right)\left(z-y_{1}\right)}{y_{2}-y_{1}} T_{z z}(z)-2 \pi \int_{y_{2}}^{\infty} \frac{\left(y_{2}-z\right)\left(z-y_{1}\right)}{y_{2}-y_{1}} T_{z z}(z) .
$$

Together with (2.30) and (2.33) this establishes (A.1) for the vacuum state.

\section{B Action of $\tilde{H}_{\text {mod on operators off the RT surface }}$}

We make the ansatz

$$
\Phi_{12}(X, T=0)=\int d k_{+} d k_{-} f\left(k_{+} k_{-}\right) e^{i\left(k_{+}-k_{-}\right) X} \mathcal{O}\left(k_{+}, k_{-}\right)
$$

where $f$ solves

$$
k_{+} k_{-} \frac{d^{2} f}{d\left(k_{+} k_{-}\right)^{2}}+2 h \frac{d f}{d k_{+} k_{-}}+\left(\left(y_{1}+y_{2}\right) X-y_{1} y_{2}-X^{2}\right) f=0
$$

This is the condition (3.33) that $\Phi_{12}$ commutes with the extended modular Hamiltonian of the segment $\left(y_{1}, y_{2}\right)$. Now consider the extended modular Hamiltonian $\tilde{H}_{\text {mod }}^{34}$ for a different

\footnotetext{
${ }^{12}$ For a general region consisting of two segments this is not a correct procedure since $f^{\prime}(z)$ vanishes somewhere in the complex plane. See the discussion section in [32]. However for the two semi-infinite segments we are considering this problem does not arise.
} 
segment $\left(y_{3}, y_{4}\right)$. We wish to compute the commutator of this new modular Hamiltonian with $\Phi_{12}$. A simple computation gives

$$
\begin{aligned}
{\left[\tilde{H}_{\text {mod }}^{34}, \Phi_{12}(Z, X, T=0)\right]=} & \frac{2 \pi i}{\left(y_{4}-y_{3}\right)}\left(\left(y_{3}+y_{4}-y_{1}-y_{2}\right) X-y_{3} y_{4}+y_{1} y_{2}\right) \times \\
& \int d k_{+} d k_{-} f\left(k_{+} k_{-}\right) i\left(k_{+}+k_{-}\right) e^{i\left(k_{+}-k_{-}\right) X} \mathcal{O}\left(k_{+}, k_{-}\right)
\end{aligned}
$$

which is just

$$
\left[\tilde{H}_{\text {mod }}^{34}, \Phi_{12}(Z, X, T=0)\right]=\frac{2 \pi i}{\left(y_{4}-y_{3}\right)}\left(\left(y_{3}+y_{4}-y_{1}-y_{2}\right) X-y_{3} y_{4}+y_{1} y_{2}\right) \partial_{T} \Phi(X, T=0)
$$

Defining $y_{4}-y_{3}=2 Z_{0}, y_{4}+y_{3}=2 X_{*}$ we get

$$
\left[\tilde{H}_{\mathrm{mod}}^{34}, \Phi_{12}(Z, X, T=0)\right]=-\frac{i \pi}{Z_{0}}\left(\left(Z_{0}^{2}-Z^{2}-\left(X-X_{*}\right)^{2}\right) \partial_{T} \Phi(Z, X, T=0)\right.
$$

which is the correct action of the bulk Rindler Hamiltonian associated with the segment $\left(X_{*}-Z_{0}, Z_{0}+X_{*}\right)$, i.e. it generates a Rindler time translation.

\section{Geodesics in BTZ}

The BTZ metric is

$$
d s^{2}=-\frac{r^{2}-r_{+}^{2}}{l^{2}} d t^{2}+\frac{l^{2}}{r^{2}-r_{+}^{2}} d r^{2}+r^{2} d \phi^{2}
$$

We look for geodesics $r(\phi)$ which extremize the action

$$
\int d \phi \sqrt{r^{2}+\frac{l^{2}}{r^{2}-r_{+}^{2}}\left(\frac{d r}{d \phi}\right)^{2}}
$$

Since nothing depends explicitly on $\phi$ there is a constant of motion which we call $r_{\min }$.

$$
r_{\min }=\frac{r^{2}}{\sqrt{r^{2}+\frac{l^{2}}{r^{2}-r_{+}^{2}}\left(\frac{d r}{d \phi}\right)^{2}}}
$$

If we choose $r\left(\phi_{0}\right)=r_{\min }$ and require $\phi(r \rightarrow \infty)= \pm L / l$ the solution after a little algebra is

$$
\frac{\cosh \frac{r_{+}}{l}\left(\phi-\phi_{0}\right)}{\cosh \frac{r_{+}}{l^{2}} L}=\sqrt{1-\frac{r_{+}^{2}}{r^{2}}}
$$

Thus two geodesics, one stretching from $-R$ to $R$ and the other from $\phi_{0}-L$ to $\phi_{0}+L$, intersect in the bulk at a point whose $\phi$ coordinate obeys

$$
\tanh \frac{r_{+}}{l} \phi=\frac{1}{\sinh \frac{r_{+}}{l} \phi_{0}}\left(\cosh \frac{r_{+}}{l} \phi_{0}-\frac{\cosh \frac{r_{+}}{l^{2}} L}{\cosh \frac{r_{+}}{l^{2}} R}\right)
$$


Open Access. This article is distributed under the terms of the Creative Commons Attribution License (CC-BY 4.0), which permits any use, distribution and reproduction in any medium, provided the original author(s) and source are credited.

\section{References}

[1] J.M. Maldacena, The Large- $N$ limit of superconformal field theories and supergravity, Adv. Theor. Math. Phys. 2 (1998) 231 [Int. J. Theor. Phys. 38 (1999) 1113] [hep-th/9711200] [INSPIRE].

[2] S. Ryu and T. Takayanagi, Holographic derivation of entanglement entropy from AdS/CFT, Phys. Rev. Lett. 96 (2006) 181602 [hep-th/0603001] [INSPIRE].

[3] V.E. Hubeny, M. Rangamani and T. Takayanagi, A Covariant holographic entanglement entropy proposal, JHEP 07 (2007) 062 [arXiv:0705.0016] [INSPIRE].

[4] T. Faulkner, M. Guica, T. Hartman, R.C. Myers and M. Van Raamsdonk, Gravitation from Entanglement in Holographic CFTs, JHEP 03 (2014) 051 [arXiv:1312.7856] [INSPIRE].

[5] I. Bena, On the construction of local fields in the bulk of $A d S_{5}$ and other spaces, Phys. Rev. D 62 (2000) 066007 [hep-th/9905186] [INSPIRE].

[6] A. Hamilton, D.N. Kabat, G. Lifschytz and D.A. Lowe, Local bulk operators in AdS/CFT: A Boundary view of horizons and locality, Phys. Rev. D 73 (2006) 086003 [hep-th/0506118] [INSPIRE].

[7] A. Hamilton, D.N. Kabat, G. Lifschytz and D.A. Lowe, Holographic representation of local bulk operators, Phys. Rev. D 74 (2006) 066009 [hep-th/0606141] [InSPIRE].

[8] A. Hamilton, D.N. Kabat, G. Lifschytz and D.A. Lowe, Local bulk operators in AdS/CFT: A Holographic description of the black hole interior, Phys. Rev. D 75 (2007) 106001 [Erratum ibid. D 75 (2007) 129902] [hep-th/0612053] [INSPIRE].

[9] T. Banks, M.R. Douglas, G.T. Horowitz and E.J. Martinec, AdS dynamics from conformal field theory, hep-th/9808016 [INSPIRE].

[10] D. Kabat, G. Lifschytz and D.A. Lowe, Constructing local bulk observables in interacting AdS/CFT, Phys. Rev. D 83 (2011) 106009 [arXiv:1102.2910] [INSPIRE].

[11] D. Kabat and G. Lifschytz, Bulk equations of motion from CFT correlators, JHEP 09 (2015) 059 [arXiv: 1505. 03755] [INSPIRE].

[12] D. Kabat and G. Lifschytz, Locality, bulk equations of motion and the conformal bootstrap, JHEP 10 (2016) 091 [arXiv: 1603.06800] [INSPIRE].

[13] I. Heemskerk, Construction of Bulk Fields with Gauge Redundancy, JHEP 09 (2012) 106 [arXiv:1201.3666] [INSPIRE].

[14] D. Kabat, G. Lifschytz, S. Roy and D. Sarkar, Holographic representation of bulk fields with spin in AdS/CFT, Phys. Rev. D 86 (2012) 026004 [arXiv:1204.0126] [INSPIRE].

[15] D. Kabat and G. Lifschytz, Decoding the hologram: Scalar fields interacting with gravity, Phys. Rev. D 89 (2014) 066010 [arXiv:1311.3020] [InSPIRE].

[16] D. Sarkar and X. Xiao, Holographic Representation of Higher Spin Gauge Fields, Phys. Rev. D 91 (2015) 086004 [arXiv:1411.4657] [INSPIRE].

[17] V.K. Dobrev, Intertwining operator realization of the AdS/CFT correspondence, Nucl. Phys. B 553 (1999) 559 [hep-th/9812194] [INSPIRE]. 
[18] N. Aizawa and V.K. Dobrev, Intertwining Operator Realization of anti de Sitter Holography, Rept. Math. Phys. 75 (2015) 179 [arXiv:1406.2129] [INSPIRE].

[19] D.L. Jafferis, A. Lewkowycz, J. Maldacena and S.J. Suh, Relative entropy equals bulk relative entropy, JHEP 06 (2016) 004 [arXiv:1512.06431] [INSPIRE].

[20] B. Czech, L. Lamprou, S. McCandlish, B. Mosk and J. Sully, A Stereoscopic Look into the Bulk, JHEP 07 (2016) 129 [arXiv: 1604.03110] [INSPIRE].

[21] B. Carneiro da Cunha and M. Guica, Exploring the BTZ bulk with boundary conformal blocks, arXiv:1604.07383 [INSPIRE].

[22] J. de Boer, F.M. Haehl, M.P. Heller and R.C. Myers, Entanglement, holography and causal diamonds, JHEP 08 (2016) 162 [arXiv:1606.03307] [INSPIRE].

[23] E. Hijano, P. Kraus, E. Perlmutter and R. Snively, Witten Diagrams Revisited: The AdS Geometry of Conformal Blocks, JHEP 01 (2016) 146 [arXiv:1508.00501] [INSPIRE].

[24] M. Miyaji, T. Numasawa, N. Shiba, T. Takayanagi and K. Watanabe, Continuous Multiscale Entanglement Renormalization Ansatz as Holographic Surface-State Correspondence, Phys. Rev. Lett. 115 (2015) 171602 [arXiv:1506.01353] [INSPIRE].

[25] H. Verlinde, Poking Holes in AdS/CFT: Bulk Fields from Boundary States, arXiv: 1505.05069 [INSPIRE].

[26] Y. Nakayama and H. Ooguri, Bulk Locality and Boundary Creating Operators, JHEP 10 (2015) 114 [arXiv: 1507.04130] [INSPIRE].

[27] H. Casini, M. Huerta and R.C. Myers, Towards a derivation of holographic entanglement entropy, JHEP 05 (2011) 036 [arXiv:1102.0440] [INSPIRE].

[28] B. Czech, L. Lamprou, S. McCandlish, B. Mosk and J. Sully, Equivalent Equations of Motion for Gravity and Entropy, JHEP 02 (2017) 004 [arXiv: 1608.06282] [INSPIRE].

[29] D.L. Jafferis and S.J. Suh, The Gravity Duals of Modular Hamiltonians, JHEP 09 (2016) 068 [arXiv: 1412.8465] [INSPIRE].

[30] T. Faulkner, R.G. Leigh, O. Parrikar and H. Wang, Modular Hamiltonians for Deformed Half-Spaces and the Averaged Null Energy Condition, JHEP 09 (2016) 038 [arXiv: 1605.08072] [INSPIRE].

[31] G. Wong, I. Klich, L.A. Pando Zayas and D. Vaman, Entanglement Temperature and Entanglement Entropy of Excited States, JHEP 12 (2013) 020 [arXiv:1305.3291] [INSPIRE].

[32] J. Cardy and E. Tonni, Entanglement hamiltonians in two-dimensional conformal field theory, J. Stat. Mech. 1612 (2016) 123103 [arXiv: 1608.01283] [INSPIRE].

[33] N. Engelhardt and G.T. Horowitz, Towards a Reconstruction of General Bulk Metrics, Class. Quant. Grav. 34 (2017) 015004 [arXiv: 1605.01070] [INSPIRE].

[34] A. Lewkowycz, G.J. Turiaci and H. Verlinde, A CFT Perspective on Gravitational Dressing and Bulk Locality, JHEP 01 (2017) 004 [arXiv: 1608.08977] [INSPIRE].

[35] M. Guica, Bulk fields from the boundary OPE, arXiv:1610.08952 [INSPIRE].

[36] W. Donnelly and S.B. Giddings, Diffeomorphism-invariant observables and their nonlocal algebra, Phys. Rev. D 93 (2016) 024030 [Erratum ibid. D 94 (2016) 029903] [arXiv: 1507.07921] [INSPIRE]. 